\title{
Increased risk of thyroid autoimmunity in rheumatoid arthritis
}

\author{
Ulla Feldt-Rasmussen ${ }^{1}$
}

Received: 30 May 2015/Accepted: 17 June 2015/Published online: 23 June 2015

(C) Springer Science+Business Media New York 2015

Two interesting papers on thyroid autoimmunity in patients with rheumatoid arthritis were recently published [1, 2]. Thyroid autoimmunity is the most common autoimmune disease, and frequently observed together with other autoimmune diseases, including rheumatoid arthritis. Both diseases are probably caused by a combination of innate and adaptive immune regulatory abnormalities, and both are diagnosed in the clinical setting by measurement of their relevant autoantibodies [thyroperoxidase (TPO) and thyrotropin receptor antibodies (TRAb), and non-organspecific autoantibodies, respectively]. Non-organ-specific antibodies have been demonstrated in up to $25 \%$ of patients with autoimmune thyroid diseases in smaller series [3], and sometimes the phenotypically clinical diseases exist in the same patients. Several previous studies of smaller series also found thyroid autoantibodies in patients with rheumatoid arthritis, but not until now has a systematic review and meta-analysis been performed [1]. The authors performed a thorough literature search and included a total of 1021 patients with rheumatoid arthritis and 1500 healthy controls. The authors only included anti-TPO and thyroglobulin antibodies (TgAb), leaving out TRAb, which are pathognomonic for Graves' disease, and extracted only 13 studies from a total of 439 articles with relevant data.

The results of the meta-analysis showed that the prevalence of thyroid autoantibody positivity in patients with rheumatoid arthritis was higher than that in healthy

Ulla Feldt-Rasmussen

ufeldt@rh.dk

1 Department of Medical Endocrinology, Rigshospital, Copenhagen University Hospital, Blegdamsvej 9, 2100 Copenhagen, Denmark controls (TgAb: OR 3.17, 95 \% CI 2.24-4.49; TPOAb: OR $2.33,95 \%$ CI 1.24-4.39). The results of this meta-analysis suggested that thyroid autoimmunity is more prevalent in patients with rheumatoid arthritis than in the control population. However, the meta-analysis does not discuss the fact that anti-TPO and TgAb are present in the majority of patients with chronic autoimmune hypothyroidism, such as Hashimoto's and atrophic thyroiditis, but that they are also present in a large proportion of patients with Graves' disease, together with TRAb. It is therefore not possible to disclose from the publication which clinical disease was more prevalent in the original studies or the meta-analysis. The general clinical impression is that Graves' disease rather than Hashimoto's thyroiditis is often seen together with rheumatoid arthritis, although this has never been documented. It is thus unfortunately not possible to predict which elevated thyroid autoimmunity risk to expect from the meta-analysis, and therefore not feasible to provide recommendations for follow-up of possible thyroid autoimmunity in patients with rheumatoid arthritis. Future studies are needed for this purpose.

A very good reason to perform such a study would be to predict induction or progression of thyroid autoimmunity using biological anti-rheumatic agents for treatment of rheumatoid arthritis, as done in the second study [2]. The authors of this publication studied prospectively 36 patients with rheumatoid arthritis treated for 3 years with Infliximab $(N=18)$ or Rituximab $(N=18)$, respectively. The patients were compared with three control groups: Twentyeight rheumatic patients treated with disease-modifying anti-rheumatic drugs and/or glucocorticoids, 21 rheumatic patients not receiving any treatment, and 49 healthy individuals. All patients had their thyroid function assessed together with thyroid autoantibodies and ultrasonography. Thyroid function was measured by serum $\mathrm{TSH}$, free $\mathrm{T} 4$ 
estimate and when relevant (suppressed TSH concentration) also T3, and thyroid autoimmunity was evidenced by anti-TPO, and TgAb, but as in the other study [1] TRAb were not measured. Ultrasonography evaluated both thyroid volume echogenicity and thyroid blood flow.

In general, thyroid function and autoantibody levels were not significantly altered at any stage irrespective of the administered biological anti-rheumatic agents, previously unknown autoimmune thyroid disease status, and/or concomitant treatment with glucocorticoids. Several other biological therapies have been shown to affect thyroid function and autoimmunity, mainly interferon- $\alpha$ [4] and Campath-1H [5]. Interferon- $\alpha$-induced thyroiditis is a prominent clinical issue in particular in patients treated for hepatitis C, usually 6-12 months after initiation of therapy. Approximately up to $15 \%$ of them will develop clinical thyroid disease and up to $40 \%$ thyroid autoantibodies [4]. The reason for these pronounced differences might well be related to diverse immune reactions such as increased activity of inflammatory cells and/or enhanced expression of class I major histocompatibility complex antigens and/or modulation of immunoglobulin production and T-helper 1 (Th1) cell predominance (4) following the use of biological anti-rheumatic agents with different modes of action.

The study also performed ultrasonography and found subtle alterations indicating possible vascular changes as early signs of thyroid autoimmune reactivity, not yet demonstrable as presence of thyroid autoantibodies. This is plausible but requires further studies.

The good news from the study is that Infliximab and Rituximab seem safely administered in patients with rheumatic diseases, including those with a concurrent newly diagnosed autoimmune thyroid disease, and screening of thyroid function and autoimmunity is not recommended as it is for e.g., interferon- $\alpha[4,6]$.
The other important conclusion from this study is that each new biological immunomodulatory agent should be tested for thyroid as well as other autoimmunity and for each disease to be treated, in order to establish concise recommendations for follow-up of each agent and each disease [7].

Conflict of interest The author declares that she has no conflict of interest.

\section{References}

1. P. Xi-Feng, G. Jian-Qiu, S. Zhong-Yan, Increased risk of thyroid autoimmunity in rheumatoid arthritis: a systematic review and meta-analysis. Endocrine (2015). doi:10.1007/s12020-015-0533-x

2. M. Kaklamanos, D. Thomas, D. Pikazis, G. Kaltsas, Thyroidspecific changes following treatment with biological therapies in patients with rheumatic diseases. Endocrine (2015). doi:10.1007/ s12020-015-0551-8

3. W.A. Scherbaum, P.Y. Youinou, P. Le Goff, G.F. Bottazzo, Antiperinuclear and rheumatoid factor in different forms of autoimmune thyroid disease. Clin. Exp. Immunol. 55, 516-518 (1984)

4. J.C. Mandac, S. Chaudhry, S.E. Sherman, Y. Tomer, The clinical and physiological spectrum of interferon-alpha induced thyroiditis:toward a new classification. Hepatology 43, 661-672 (2006)

5. A.J. Coles, M. Wing, S. Smith, F. Coraddu, S. Greer, C. Taylor, Pulsed monoclonal antibody treatment and autoimmune thyroid disease in multiple sclerosis. Lancet 354, 1691-1695 (1995). doi:10.1016/S0140-6736(99)02429-0

6. A. Hauschild, H. Gogas, A. Tarhini, M.R. Middleton, A. Testor, B. Dreno et al., Practical guidelines for the management of interferon$\alpha-2 b$ side effects in patients receiving adjuvant treatment for melanoma. Cancer 112, 982-994 (2008)

7. F. Torino, A. Barnabei, R.M. Paragliola, P. Marchetti, R. Salvatori, S.M. Corsello, Endocrine side-effects of anti-cancer drugs: mAbs and pituitary dysfunction: clinical evidence and pathogenic hypotheses. Eur. J. Endocrinol. 169, 153-164 (2013). doi:10. 1530/EJE-13-0434 